\title{
Metacognitive Feelings, Conflict Detection and Illusion of Linearity
}

\author{
Vanja Putarek and Vesna Vlahović-Štetić \\ University of Zagreb, Faculty of Humanities and Social Scences, \\ Department of Psychology, Zagreb, Croatia
}

\begin{abstract}
Studies on conflict detection have suggested that people are sensitive to conflict between their heuristic judgment and logical or probabilistic principles, but due to the inhibition failure, they do not disregard appealing heuristic answer. However, these studies were mostly conducted on syllogistic reasoning and base-rate problems. The question is whether findings about conflict detection can be applied to other materials and areas of reasoning. Current study focused on the illusion of linearity, in which people over-rely on linearity heuristic. Therefore, the aim of this study was to examine whether students detect the conflict between heuristic answer and logical/mathematical principles. Participants were 113 secondary school students from Zagreb, Croatia. Data were collected using a computer program, which consisted of instructions, 20 problems (10 linear and 10 non-linear) and sociodemographic questions. Problems were presented randomly in multiple-choice format and had three offered answers (correct answer, distractor, "none of the answers"). Response time for each problem was also measured. Results demonstrated that students mostly solved non-linear problems incorrectly and in accordance with linearity heuristic. Furthermore, the analysis of metacognitive feelings of confidence and difficulty revealed that students detected conflict between heuristic answer and mathematical principles. Moreover, overriding heuristic answer and generating correct answer to non-linear problems resulted in increased response time. Comparison between metacognitive feelings and response time in linear and non-linear problems indicates the importance of processing fluency and inhibition failure in the occurrence of the illusion of linearity.
\end{abstract}

Keywords: the illusion of linearity, metacognitive feelings, conflict detection, dual-process theories

Vanja Putarek, Faculty of Humanities and Social Sciences, University of Zagreb, Ivana Lučića 3, 10000 Zagreb, Croatia. E-mail: vputarek@ffzg.hr 


\section{Introduction}

\section{The Illusion of Linearity}

Linearity or proportionality is omnipresent in everyday life and represents one of the key concepts in various mathematical fields, such as algebra, statistics, and vectors (De Bock, Van Dooren, Janssens, \& Verschaffel, 2007; Van Dooren, De Bock, Janssens, \& Verschaffel, 2008). Consequently, by increasing the knowledge and usage of linearity throughout formal and informal education, numerous students tend to apply linear model universally. This may lead to error known as the illusion of linearity or proportionality, a propensity to comprehend certain sizes as linearly or proportionally related, even in situations where such understanding is not justified (De Bock et al., 2007).

Although linear relations are more intuitive than non-linear ones, young children have an implicit understanding of non-linearity, based on informal daily experiences. Namely, research revealed that 5-year old children successfully differentiate between growth situations that developed either in a linear or non-linear manner (Ebersbach, Van Dooren, Goudriaan, \& Verschaffel, 2010). Despite basic and implicit understanding of non-linearity, linearity principle prevails over nonlinearity principle because during elementary school students use linear functions more often than non-linear functions and linear models are reinforced. The erroneous usage of linearity continues in secondary school and university, as well as in adults (De Bock et al., 2007; Esteley, Villarreal, \& Alagia, 2010).

Well-known examples of this error are observed in geometry problems in which enlargement/reduction with factor $k$, enlarges/reduces the area, not with factor $k$, but with factor $k^{2}$, and volume with factor $k^{3}$. For instance, $2 \%$ of 12 - to 13 -year-olds and $17 \%$ of 15- to 16-year-olds solved correctly problems such as "Farmer Carl needs 6 bags of grass seed to cover a square pasture with a side of 200 meters. How many bags of grass seed will he need to cover a square pasture with a side of 600 meters?" (54 bags), while more than $80 \%$ gave incorrect linear answer (18 bags) (De Bock, Verschaffel, \& Janssens, 1998).

The resistance of the illusion of linearity was found in studies that used different methods to decrease this error. When students were solving problems with direct area measures, they were not more successful than students who were solving problems with indirect measures (Van Dooren, De Bock, De Bolle, Janssens, \& Verschaffel, 2003). De Bock, Verschaffel, and Janssens (2002) used metacognitive and visual scaffolds to influence students' noticing of non-linearity, which decreased the illusion of linearity, but effects were small. While researchers usually used problems in an open-ended format, Vlahović-Štetić, Pavlin-Bernardić, and Rajter (2010) used problems in multiple-choice format with five offered answers. When linear answer was not offered in non-linear problems, students were more successful than students 
who had linear answer, but students' performance in linear problems was better than performance in non-linear problems.

According to De Bock, Van Dooren, Janssens, and Verschaffel (2002), the illusion of linearity is based on the intuitiveness of linear model, shortcomings in geometrical knowledge, poor use of mathematical useful heuristics, and maladaptive habits and beliefs towards mathematical word problem-solving. Furthermore, linear reasoning is heuristic-based (Gillard, Van Dooren, Schaeken, \& Verschaffel, 2009). Namely, during elementary school, linear model is frequently used, which strengthens a linearity scheme that starts to be rapidly and easily activated in various situations and consequently leads to the formation of linearity heuristic. Contrary to that, non-linear reasoning requires deep and analytic processes. Distinction between automatic and analytic processes is central aspect of dual-process theories, which can be used as a framework for better understanding of the illusion of linearity.

\section{Dual-Process Theories and Conflict Detection}

Dual process theories are focused on two ways of information processing, labelled as Type 1 and Type 2 . Type 1 processing is intuitive or heuristic, occurs automatically and engages minimal working memory. It is fast, effortless, inflexible, not influenced by verbal instructions, and tends to solve problems relying on prior knowledge and beliefs (Stanovich \& Toplak, 2012). Type 2 processing is slow and requires cognitive effort, thus engaging working memory. It is controlled, flexible, evolutionary younger, can be verbally instructed, and is based on conscious thinking.

These two types of processing often work in agreement (De Neys, 2012; Evans $\&$ Stanovich, 2013). In these situations Type 1 processing generates correct answer, which Type 2 processing does not have to scrutinize, so automatic and analytic answer are congruent and logically/normatively correct. Besides congruent situations, automatic answer and logical principles can be in conflict, that is, Type 1 processing generates a biased answer and analytic Type 2 processing needs to override and inhibit this answer in order to provide normatively correct answer. In these conflicting situations automatic and analytic answer are incongruent. For instance, in heuristic and biases problems adapted from classical studies of Kahneman and Tversky, individuals' first answer generated by Type 1 processing usually is heuristic (e.g., representativeness heuristic), and this biased and heuristicbased answer is not in accordance with correct answer that Type 2 processing can provide (De Neys, 2014). Accuracy in these incongruent situations is usually quite low (De Neys \& Glumicic, 2008; Kahneman \& Frederick, 2002), which means that people are not good at inhibiting first and biased answer, as well as that biased answers may be more compelling than analytic answers. However, the research on the conflict detection indicated that, although people usually do not inhibit heuristic answer and do not manage to verbalize the exact normative principles that are being violated, they detect conflict between heuristic answer and logical principles. 
Namely, in congruent situations response time is faster than in incongruent situations (Bonner \& Newell, 2010; De Neys \& Glumicic, 2008). The slowest response time was obtained when people provide correct answer in incongruent situations, but even people who gave heuristic answer in incongruent situations spent more time processing these problems compared to situations when they were solving congruent problems. That is, people process incongruent problems less fluently than congruent problems. De Neys, Moyens, and Vansteenwegen (2010) also showed that in incongruent situations autonomous nervous system (measured via skin conductance answers) is aroused, while this arousal is absent in congruent situations. It can be concluded that, although answers in congruent situations are often biased, people have a "gut feeling" that signals them that they are wrong (De Neys, 2010).

In the context of the illusion of linearity, Gillard et al. (2009) confirmed that linear answers have heuristic characteristics. Namely, in that study, when response time was restricted and executive resources were burdened with a secondary problem, in non-linear problems the frequency of linear answers increased, while the frequency of correct answers decreased. That is, Type 2 processing could not override automatic answer generated by Type 1 processing. As mentioned above, linear answers could become automatic because linear schema is more intuitive than non-linear schema and because mathematics in elementary school is mostly based on linearity (De Bock et al., 2002). Therefore, it can be assumed that non-linear problems represent incongruent situations, in which linear answer is biased and heuristic-based, while non-linear answer is analytic answer that is normatively correct. According to the research on conflict detection, response time should be slower and confidence for biased answer in non-linear (i.e., incongruent) situations should be lower than for correct answers in linear (i.e., congruent) situations.

These assumptions were not examined yet, but the findings about the underlying mechanisms of the illusion of linearity could help researchers and practitioners to understand mathematical reasoning, and find adequate interventions to decrease this illusion. In order to accomplish these goals, the first important step is to examine the differences in processing of correct and incorrect answers in linear and non-linear problems. One by-product of information processing are metacognitive experiences, so in the present study we will examine the differences in metacognitive experiences between answers in linear and non-linear problems.

\section{Metacognitive Experiences}

Metacognition refers to the cognition of cognition, and infers about cognition through monitoring and modifies cognition through control (Flavell, 1979; Nelson \& Narens, 1994). Metacognitive processes that are included in the monitoring and control of reasoning and problem solving are labelled as meta-reasoning (Ackerman $\&$ Thompson, 2017). When a person needs to decide whether he or she would engage metacognitive control and consequently change cognitive strategy, metacognitive 
experiences have the crucial role (Efklides, 2009, 2014). Metacognitive experiences represent experiential feelings (e.g., feeling of confidence, difficulty, knowing, familiarity) and judgments (e.g., judgment of learning, estimation of time or effort) that inform a person about cognitive processing, and serve as the interface between the problem and the person (Efklides, 2006).

Although factors such as individuals' pre-existing beliefs about information processing (Frank \& Kuhlmann, 2017; Mueller, Dunlosky, \& Tauber, 2016) or explicit problem demand (Song \& Schwarz, 2008) have an important role in forming metacognitive experiences, the primary sources are nonanalytic, non-conscious inferential processes (Norman, Price, \& Duff, 2010). It is assumed that during information processing its by-products are generated and they serve as cues for the appearance of metacognitive experiences. The crucial cue is the fluency of information processing (Koriat, Bjork, Sheffer, \& Bar, 2004), which represents the subjective experience of the ease of information processing (Reber \& Greifeneder, 2017) and may take different forms, such as perceptual, conceptual or linguistic fluency (Alter \& Oppenheimer, 2009). Some authors termed the dependence of metacognitive experiences on fluency as the fluency heuristic (Hertwig, Herzog, Schooler, \& Reimer, 2008).

For instance, when a person encounters previously processed information, this information would be fluently processed and feeling of familiarity (FOF) would arise, associated with positive affect (Efklides, 2006). Feeling of difficulty (FOD) is associated with negative affect (Efklides, Samara, \& Petropoulou, 1999). In familiar problems, FOD increases due to the working memory load, as well as due to the lack of fluency that arises when conflict between two simultaneously activated answers appears (Touroutoglou \& Efklides, 2010). In less familiar problems, FOD increases with cognitive interruptions, that is, when individuals notice that their schemas cannot be applied to new information. FOD and FOF, jointly with the estimate of answer correctness, determine feeling of confidence (FOCon) (Efklides, 2002; Shynkaruk \& Thompson, 2006), which is related to the outcome of processing (Narens, Jameson, \& Lee, 1994). Metacognitive experiences are interrelated, that is, higher FOF is positively related to FOCon, while both FOF and FOCon are negatively related to FOD (Efklides et al., 1999). These correlations were low to mediate, which justifies the differentiation of these experiences.

According to the meta-reasoning framework (Ackerman \& Thompson, 2017), the quality of metacognitive experiences determines how Type 2 processing would be engaged. The researchers usually explored feeling of rightness (FOR) (e.g., Thompson, Prowse Turner, \& Pennycook, 2011), which is similar to the previously described FOCon, but it is estimated for the first, automatic answer and represents strong intuition that this answer is correct (Thompson, 2009). In order to verify this theory, researchers used two-response paradigm and asked participants to give their first, automatic or intuitive answer to reasoning problem, estimate FOR, and then the same problem was presented and participants could take as much time as they wanted 
to give final answer (Thompson et al., 2011). It was revealed that participants who had strong FOR were less inclined to change their answer when the problem was presented second time, regardless of the normative accuracy of their first answer.

Experiences are always present as online or concurrent metacognition during information processing and produce cues about the progress of this processing (Efklides, 2006). Therefore, they can inform people about the conflict between heuristic answer and logical principles. This assumption was confirmed in several studies. Simmons and Nelson (2006) demonstrated that FOCon depends on the intuitiveness of answers and it is higher for intuitive answers than for equally valid, but non-intuitive answers. Study conducted by De Neys, Cromheeke, and Osman (2011; Experiment 1) demonstrated that participants were less confident (lower FOCon) in their heuristic and incorrect answers in incongruent situations than in correct answers in incongruent situations and correct answers in congruent situations, but they were equally confident in correct answers in incongruent situations and congruent situations. That is, participants detected conflict, which was manifested in their metacognitive experiences.

\section{Current Study}

The research on two types of processing and conflict detection has been conducted mostly on syllogistic reasoning or probability estimation problems from heuristics and biases studies (e.g., Thompson et al., 2013). The question is whether the findings from these studies can be applied to problems that can be found in reallife and formal education more often than abstract syllogisms or probability estimation problems. The illusion of linearity includes problems that are prone to intuitive answers, but at the same time, people acquire knowledge for their solving during formal education. Namely, linear and non-linear reasoning are widespread in mathematics and they represent the basis of numerous mathematical fields (De Bock et al., 2007). They are also embedded in real-life situations, for instance, making financial decisions and understanding of everyday phenomena, such as weather, time or temperature. Due to the omnipresence of linear and non-linear reasoning, as well as the importance of mathematics for overall academic achievement and everyday life (Lerkkanen, Rasku-Puttonen, Aunola, \& Nurmi, 2005; Rose \& Betts, 2001), it would be important to comprehend how over-reliance on linear model is maintained, and afterwards determine the methods aimed at the decreasing this illusion. According to the literature available to date, the researchers did not focus on conflict detection in the context of the illusion of linearity. Therefore, the aim of this study was to examine whether students detect the conflict between heuristic answer and logical or mathematical principles in non-linear problems. The criteria for conflict detection were the levels of metacognitive experiences and response time.

Given that the illusion of linearity is widespread and deep-rooted phenomena (De Bock et al., 1998; Van Dooren et al., 2003), we assumed that in our sample of 
15- to 18-year old adolescents the illusion of linearity would be obtained. The research conducted by Vlahović-Štetić et al. (2010) revealed that students are prone to the illusion of linearity even when problems are shown in multiple-choice format. Therefore, in our research we presented problems in multiple-choice format, which are less time-consuming. We offered three answers in both linear and non-linear problems: correct answer, distractor (in non-linear problems distractor represented linear answer to non-linear problem), and answer "none of the answers". The third answer, "none of the answers", was offered in order to decrease students' guessing.

According to the research on conflict detection and monitoring, as well as dualprocess theories (e.g., De Neys \& Glumicic, 2008; Gillard et al., 2009), it can be assumed that linear problems represent congruent problems (i.e., heuristic answer is normatively correct) and non-linear problems represent incongruent problems (i.e., heuristic answer is not normatively correct and it is in conflict with mathematical principles). We expected that metacognitive experiences would differ between answers in linear and non-linear problems. More precisely, in the present study, the focus was on four metacognitive feelings: feeling of confidence, difficulty, familiarity, and comprehensibility. Feelings such as FOCon, FOD and FOF were previously described, and relations between them were explored (e.g., Efklides et al., 1999). In the context of mathematical problem solving, students' performance can be low, not because of the shortcomings in their knowledge or perceived problem difficulty, but due to the problem wording and comprehensibility (Verschaffel, Greer, \& De Corte, 2000). Therefore, we included the feeling of comprehensibility (FOCom) that could represent students' perception of problem-wording.

Given the findings that participants had lower FOCon in their heuristic answers in incongruent situations than in correct answers in congruent situations (De Neys et al., 2011), we assumed that FOCon, FOF and FOCom would be lower, while FOD higher, for linear (i.e., heuristic and normatively incorrect) answers in non-linear problems than linear (i.e., normatively correct) answers in linear problems. That is, students would detect conflict, which would decrease fluency of information processing, and this conflict detection would be manifested in metacognitive experiences. When students solve incongruent problems correctly, they detect conflict and override it because they have available alternative cognitive schema (De Neys et al., 2011). However, when students detect conflict and do not have available alternative cognitive schema, they are "stuck" with their heuristic answer, although they are aware that this answer is wrong. Therefore, we assumed that FOCon, FOF and FOCom would be higher, while FOD lower, for non-linear (i.e., normatively correct) answers in non-linear problems than for heuristic answers in non-linear problems.

We also measured how much time students would need to answer the problem. Previous research indicated that individuals respond faster in congruent than in incongruent situations (e.g., Bonner \& Newell, 2010). Therefore, we assumed that students would respond faster when they produce correct answer in linear problems 
than when they produce heuristic answer in non-linear problems, and that response time would be the slowest for generating correct answers in non-linear problems because students engage in finding alternative cognitive schema (De Neys \& Glumicic, 2008).

Finally, we examined the metacognitive experiences and response time when the third offered answer (i.e., "none of the answers") was selected. Previous research on conflict detection (e.g., De Neys \& Glumicic, 2008) used problems with two offered answers (in congruent problems: distractor and correct answer; in incongruent problems: heuristic answer and correct answer). However, research in the context of the Diminishing Criterion Model (Ackerman, 2014) demonstrated that people tend to give answers, which satisfy their confidence criterion. When people perceive that they invested an immense amount of time and reach the effort limit, but did not meet confidence criterion, they are inclined to select "don't know" answer. In line with these assumptions, Ackerman (2014; Experiment 4 and 5) obtained that response time for "don't know" answers was longer than for correct or incorrect answers.

We hypothesized that students who selected "none of the answers" would behave similarly to participants who selected "don't' know" answer in Ackerman's (2014) research. That is, students, who selected "none of the answers", would invest time and effort in searching for correct answer, but that answer was not available. Consequently, they gave up and used "none of the answers" as an exit strategy to complete the problem and move on the next problem. Therefore, we assumed that FOCon, FOF, and FOCom would be lower, FOD higher, and response time slower when students selected "none of the answers" than when they selected correct answers and distractors.

\section{Method}

\section{Participants}

The participants were 113 students (55\% female) from one academic-track secondary school in Zagreb, Croatia. Students' age ranged from 15 to $18(M=15.85$, $S D=0.66)$. There were $89(80.2 \%)$ second-grade and $22(19.8 \%)$ third-grade students (two students did not report their grade).

\section{Materials}

Students solved 10 linear and 10 non-linear problems. Following each problem, students were asked to rate their level of confidence that their answer is correct, using the scale from 1 (not at all) to 7 (highly confident). Students also estimated how difficult (FOD), familiar (FOF) and comprehensible (FOCom) was the problem, 
using the scale from 1 (not at all) to 5 (highly difficult/familiar/comprehensible). At the end of the study, for each student, the sum of correct answers was presented on a computer screen.

Example of linear problem:

In order to set up the fence around the square-shaped playground with a side of $60 \mathrm{~m}$, workers need 3 days. How much time workers need to set the fence around the square-shaped playground with a side of $120 \mathrm{~m}$ ? The speed of work is the same in both cases.

a) 9 days

b) 6 days (correct answer)

c) none of the answers

Example of non-linear problem:

There are 10 apple trees in the square-shaped orchard with a side of $5 \mathrm{~m}$. How many apple trees can grow in a square-shaped orchard with a side of $10 \mathrm{~m}$ ? The distance between the apple trees is the same in both orchards.

a) 40 apple trees (correct answer)

b) 20 apple trees

c) none of the answers

\section{Procedure}

For this study, the computer program was developed, which contained the instruction, 20 problems, sociodemographic questions, and questions about mathematic achievement. At the beginning of the assessment, the instructions on the computer screen were presented, followed by 10 linear and 10 non-linear problems (randomly displayed). For each problem three answers were offered: correct answer, distractor (in non-linear problems distractor represented linear answer to non-linear problem), and answer "none of the answers". Correct answer and distractor were presented randomly as a first or second offered answer, while "none of the answers" was always presented as the third offered answer. Problems were presented in two steps: a problem without answers was presented and reading time was measured, and when students read the problem, they pressed "next" button and three answers appeared (text of the problem remained on the screen), and solving time was measured.

Data were collected in the autumn of the school year. Permission for conducting this research was granted by school principals and the Ethical Committee of the Department of Psychology Faculty of Humanities and Social Studies, University of Zagreb, Croatia. The researcher informed students that all data will be collected anonymously and that they are allowed to terminate participation at any time during the assessment. After a brief introduction, all students agreed to participate. Students 
were told that all information on how to solve the problems will be presented on the computer screen and they were asked to read very carefully the instructions. It took students 25 to 45 minutes to solve problems on computers in classes.

\section{Results}

\section{Reasoning Accuracy}

On average, students solved $11.25(S D=2.87$; range: 5-19) problems correctly. In linear problems, 46 students $(40.7 \%)$ solved all 10 problems correctly and a minimal number of correct answers was 3 . In non-linear problems, 4 students $(3.8 \%)$ solved all 10 problems correctly and 39 students $(37.5 \%)$ did not solve any of 10 problems correctly. The mean number of correct answers, distractors and "none of the answers" in linear and non-linear problems is presented in Table 1.

Table 1

The Average Number of Correct Answers, Distractors and "None of the Answers" in Linear and Non-Linear Problems $(N=104)$

\begin{tabular}{lcccc}
\hline Type of problem & & Correct answer & Distractor & "None of the answers" \\
\hline \multirow{2}{*}{ Linear } & $M$ & 8.73 & 0.58 & 0.69 \\
& $S D$ & 1.60 & 1.07 & 1.21 \\
\hline \multirow{2}{*}{ Non-linear } & $M$ & 2.52 & 6.86 & 0.63 \\
& $S D$ & 2.95 & 3.14 & 1.10 \\
\hline
\end{tabular}

Students solved more linear than non-linear problems correctly $(t(103)=16.78$, $p<.001)$. Repeated-measures ANOVA with Bonferroni correction revealed that in linear problems students selected correct answer more often than distractor and "none of the answers", and they selected distractor as often as "none of the answers" $\left(F(1.60,164.43)=880.02, p<.001, \eta_{\mathrm{p}}^{2}=.90\right)$. In non-linear problems students selected distractor (heuristic) answer more often than correct answer and "none of the answers", and correct answer more often than "none of the answers" $\left(F(1.19,122.87)=107.61, p<.001, \eta_{\mathrm{p}}^{2}=.51\right)$.

\section{Metacognitive Feelings, Response Time, and Conflict Detection}

In order to examine conflict detection, average ratings of metacognitive feelings and response time for each student for three offered answers (correct answer, distractor, "none of the answers") were calculated. Afterwards, we computed repeated-measures ANOVA to compare differences in metacognitive feelings and response time between metacognitive feelings for these three offered answers. In analyses were included only students who had at least one correct answer in linear 
and non-linear problems and selected at least one distractor in non-linear problems. Descriptive statistics and the results of ANOVA are presented in Table 2.

Table 2

Descriptive Statistics of Metacognitive Feelings for Correct Answers in Linear and NonLinear Problems, as Well as Distractors (Heuristic Answers) in Non-Linear Problems $(N=$ 60)

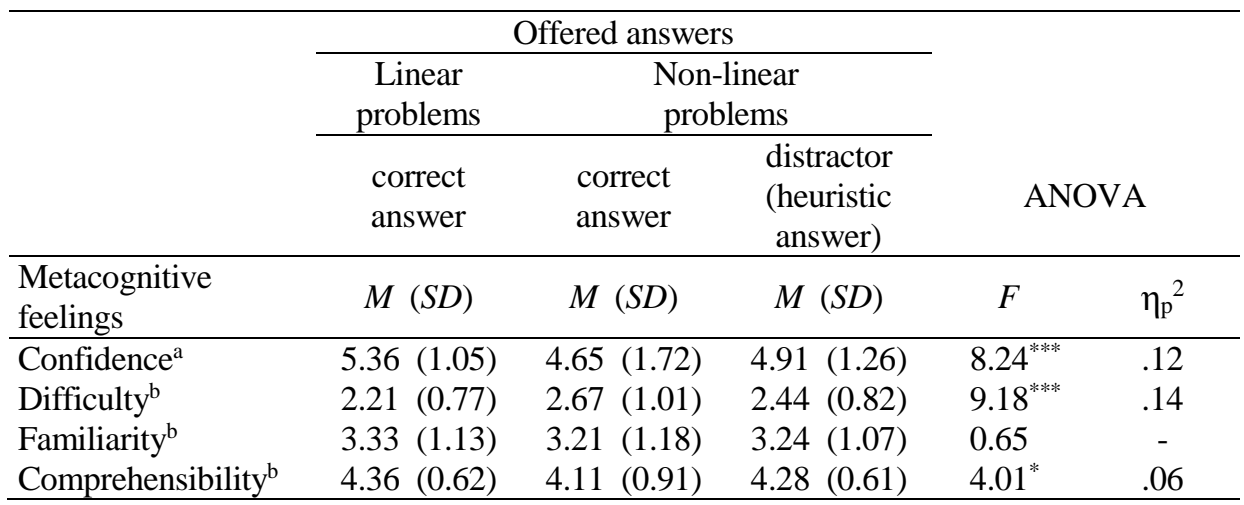

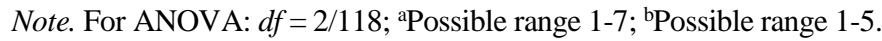

${ }^{*} p<.05 ;{ }^{* * *} p \leq .001$.

Post-hoc comparison with Bonferroni correction demonstrated that FOCon was higher for correct answers in linear problems compared to correct answers in nonlinear problems, and heuristic answers in non-linear problems, while there were no differences in FOCon between correct and heuristic answers in non-linear problems.

Students had lower FOD for linear problems in which they selected correct answer than for non-linear problems in which they selected correct answer and nonlinear problems in which they selected heuristic answer. There were no differences in FOD between correct and heuristic answers in non-linear problems.

There were no differences in FOF between correct answers in linear problems, correct answers, as well as heuristics answers in non-linear problems. FOCom was boundary higher for correctly solved linear problems than correctly solved non-linear problems $(p=.065)$. There were no differences in FOCom for linear problems in which students selected correct answer and non-linear problems in which they selected heuristic answer, as well as between correct answers and heuristic answers in non-linear problems.

In regards to response time, in linear problems, average reading time was 29.40 $\sec (S D=16.96)$ and it was longer than average solving time $(M=13.02, S D=11.09)$ $(t(103)=7.49, p<.001)$. Similarly, in non-linear problems, average reading time was $30.06 \mathrm{sec}(S D=15.46)$ and it was longer than average solving time $(M=14.59, S D$ $=11.46)(t(103)=7.49, p<.001)$. It seems that students were solving problems 
during reading, and when answers appeared, only searched for their answer. Therefore, it was difficult to divide reading and solving time, so their sum was used to assess response time. On average, students spent $43.82 \mathrm{sec}$ per problem $(S D=$ 15.63, range 15.21-87.21; $C=41.53$ ). All response time (RT) measures were converted to $\log 10$ prior to analysis.

Repeated measures ANOVA $\left(F(2,118)=6.53, p=.002, \eta_{\mathrm{p}}^{2}=.10\right)$ demonstrated that RT did not differ between linear problems in which correct answers were selected and non-linear problems in which heuristic answers were selected, but it was longer when correct answers in non-linear problems were selected than when correct answers in linear problems were selected. There were no differences in RT between correct and heuristic answers in non-linear problems.

\section{Metacognitive Feelings, Response Time, and the Type of Answer}

There were 16 students in linear problems and 24 students in non-linear problems who at least once selected correct answer, distractor, and "none of the answers". Therefore, comparison of metacognitive feelings between selected answers was computed for the small subsample. Descriptive statistics and the results of Friedman test for testing differences in metacognitive feelings between offered answers are presented in Table 3.

Table 3

Descriptive Statistics of Metacognitive Feelings for Correct Answers, Distractors and "None of the Answers", and the Results of Friedman Test, as Well as Results of Post Hoc Test, for Differences Between Them, in Linear $(N=16)$ and Non-Linear $(N=24)$ Problems

\begin{tabular}{|c|c|c|c|c|c|c|c|}
\hline \multirow[b]{3}{*}{$\begin{array}{l}\text { Metacognitive } \\
\text { feelings }\end{array}$} & \multirow[b]{3}{*}{$\begin{array}{l}\text { Type of } \\
\text { problem }\end{array}$} & \multicolumn{3}{|c|}{$\begin{array}{c}\text { Offered answers } \\
\end{array}$} & \multirow{3}{*}{$\begin{array}{c}\text { Friedman } \\
\chi^{2}\end{array}$} & \multirow{2}{*}{\multicolumn{2}{|c|}{$\begin{array}{l}\text { Post-hoc test } \\
\text { (Wilcoxon Z) }\end{array}$}} \\
\hline & & \multirow{2}{*}{$\begin{array}{c}\begin{array}{c}\text { Correct } \\
\text { answer (1) }\end{array} \\
M(S D)\end{array}$} & \multirow{2}{*}{$\begin{array}{c}\text { Distractor } \\
(2)\end{array}$} & \multirow{2}{*}{$\begin{array}{c}\begin{array}{c}\text { None of the } \\
\text { answers (3) }\end{array} \\
M(S D)\end{array}$} & & & \\
\hline & & & & & & $1-3$ & $2-3$ \\
\hline \multirow{2}{*}{ Confidence $^{\mathrm{a}}$} & Linear & $4.97(0.82)$ & $4.15(1.18)$ & $3.31(1.86)$ & $13.74^{* * * *}$ & $2.97^{* * *}$ & 1.77 \\
\hline & Non-linear & $4.59(1.54)$ & $4.72(0.89)$ & $3.73(1.08)$ & $7.02^{*}$ & $2.02^{*}$ & $2.95^{* *}$ \\
\hline \multirow{2}{*}{ Difficulty $^{\mathrm{b}}$} & Linear & $2.53(0.75)$ & $2.88(0.81)$ & $3.26(0.69)$ & 4.26 & - & - \\
\hline & Non-linear & $2.72(0.90)$ & $2.53(0.71)$ & $3.35(0.75)$ & $13.13^{* * *}$ & $2.49^{* *}$ & $3.53^{* * * *}$ \\
\hline \multirow{2}{*}{ Familiarity ${ }^{\mathrm{b}}$} & Linear & $3.37(1.12)$ & $3.62(1.26)$ & $2.90(1.30)$ & $9.41^{* *}$ & $1.99^{*}$ & $2.24^{*}$ \\
\hline & Non-linear & $3.28(1.11)$ & $3.42(0.97)$ & $2.81(1.15)$ & $6.49^{*}$ & $1.90^{\dagger}$ & $2.75^{* *}$ \\
\hline \multirow{2}{*}{ Comprehensibility ${ }^{\mathrm{b}}$} & Linear & $4.41(0.56)$ & $4.06(0.98)$ & $3.46(1.13)$ & $9.64^{* * *}$ & $2.76^{* *}$ & 1.68 \\
\hline & Non-linear & $4.23(0.69)$ & $4.09(0.69)$ & $3.47(0.91)$ & $13.93^{* * * *}$ & $3.76^{* * * *}$ & $2.89^{* * *}$ \\
\hline
\end{tabular}

Note. For Friedman test: $d f=2$; a Possible range 1-7; bossible range 1-5.

$\dagger .06 ;{ }^{*} p<.05 ;{ }^{* *} p \leq .01 ; * * * 0.001$.

In both linear and non-linear problems, FOCon was higher for correct answers than for "none of the answers". On the one hand, in linear problems, there were no 
differences in FOCon for distractors and "none of the answers". On the other hand, in non-linear problems, FOCon was higher for heuristic answers compared to "none of the answers".

Friedman test did not reveal any differences in FOD in linear problems when correct answer, distractor and "none of the answers" were selected. In non-linear problems, problems' difficulty was higher when students selected "none of the answers" than when they selected correct answer or heuristic answer.

With regards to FOF, in both linear and non-linear problems this feeling was lower when students selected "none of the answers" than when they selected distractor or correct answer (in non-linear problems, difference in FOF when students selected correct answer and "none of the answers" was at the boundary level of significance).

In both linear and non-linear problems, FOCom was higher for problems in which correct answer was selected than for problems in which "none of the answers" was selected. While in linear problems there were no differences in FOCom for problems in which distractor and problems in which "none of the answers" were selected, in non-linear problems in which "none of the answers" was selected were estimated as less comprehensible than non-linear problems in which heuristic answer was selected.

In regards to RT, Friedman test was significant for linear problems $\left(\chi^{2}(2)=9.56\right.$, $p=.01)$ and non-linear problems $\left(\chi^{2}(2)=6.33, p=.04\right)$. In linear problems, RT was longer when "none of the answers" was selected than when correct answer (Wilcoxon $Z=2.64, p=.01$ ) or distractor (Wilcoxon $Z=2.67, p=.01$ ) were selected. Similarly, in non-linear problems, RT was longer when "none of the answers" was selected than when heuristic answer was selected (Wilcoxon $Z=2.52, p=.01$ ), while the difference in RT was at the boundary level when "none of the answers" was selected and when correct answer was selected (Wilcoxon $Z=1.97, p=.051$ ), that is, for "none of the answers" RT was slower than for correct answers.

\section{Discussion}

The aim of the present study was to examine the presence of conflict detection in the illusion of linearity. Conflict detection was measured via metacognitive experiences (FOCon, FOD, FOD and FOCom), as well as response time. Previous research repeatedly obtained that people use linear model to solve various problems and apply it regardless of its appropriateness, which is called the illusion of linearity (De Bock et al., 1998). In our study, students were inclined to select linear answers in non-linear problems and they solved linear problems more accurately than nonlinear problems. Hence, our study confirms the illusion of linearity among 15- to 18year old adolescents and when multiple-choice format with three answers was used. 
It seems that students behave as cognitive misers, regardless of the type of problem format (i.e., open-ended or multiple-choice) and the number of offered answers.

\section{Metacognitive Experiences, Response Time, and Conflict Detection}

In order to decrease the presence of the illusion of linearity, it is important to understand how this misleading thinking is formed and maintained. Up to date, researchers were mostly focused on its formation and it was found that the illusion of linearity is largely developed during elementary school and that it is difficult to overcome appealing linear answer (De Bock et al., 2007). Current study revealed additional findings related to conflict detection that can help us to understand how the illusion of linearity functions, and consequently how it is maintained.

Conflict detection studies used congruent problems, in which automatic answer is normatively correct, and incongruent problems, in which automatic answer is heuristic and normatively incorrect (e.g., De Neys \& Glumicic, 2008; De Neys et al., $2010,2011)$. Automatic answer is generated by Type 1 processing and in congruent problems Type 2 processing does not have to analyse it (De Neys, 2012). However, in order to respond correctly in incongruent problems, people have to engage analytic Type 2 processing, inhibit automatic (i.e., heuristic and normatively incorrect) answer, and override it.

Given the results of previous studies, which indicated that it is difficult to overcome the linear answer in non-linear problems (e.g., De Bock et al., 2002), as well as that linear answer has heuristic characteristics (Gillard et al., 2009), in present study linear problems were defined as congruent problems, while non-linear problems represent incongruent problems.

Our results confirmed the assumption that students detect conflict between heuristic answer and logical/mathematical principles in non-linear problems. Namely, FOCon was higher for correct answers in linear problems than for heuristic answers in non-linear problems, although linear and non-linear problems, in which these answers were selected, were perceived as equally comprehensible (i.e., there were no differences in FOCom between them). It is important to note that heuristic answer in non-linear (i.e., incongruent) problems and correct answer in linear (i.e., congruent) problems were both linear answers. Metacognitive experiences represent conscious manifestations of non-conscious cues, such as the fluency of information processing (Efklides, 2006; Koriat et al., 2004). For instance, when information is less fluently processed due to the problems' perceived difficulty, unfamiliarity, or some other obstacles during processing, individuals are less confident in their answers or estimate these problems as more difficult compared to problems that are fluently processed. Consequently, conflict detection can be manifested in the level of metacognitive experiences. That is, when conflict is detected, problem-solving is interrupted and information processing does not proceed fluently, so FOCon are lower. 
Our finding is in accordance with previous research on conflict detection, which indicated that individuals are more confident in their correct answers in congruent problems than in heuristic answers in incongruent problems (De Neys et al., 2011). Therefore, the conclusion of conflict detection studies that people detect conflicts between heuristic answers and logical principles can be applied to the illusion of linearity. It seems that students have "gut feeling" that they are wrong, but due to the inhibition failure, they do not disregard heuristic answer (De Neys \& Glumicic, 2008). In the context of the illusion of linearity, it is possible that the inhibition failure arises because of poor mathematical knowledge and unavailability of alternative cognitive schema. Regardless of this possible mathematical ignorance, we can conclude that students "felt" the difference between linear and non-linear problems.

In line with these findings are the results of FOD. Namely, students perceived non-linear problems in which they selected heuristic answer as more difficult than linear problems in which they selected correct answers. As was assumed for FOCon, it seems that students actually analysed non-linear problems and detected conflict between heuristic answer and logical/mathematical principles, which could result in cognitive interruption (Touroutoglou \& Efklides, 2010). That is, they noticed that their linear schema could not be applied to non-linear problems, but it seems that alternative cognitive schema and inhibitory processes were not available to them, so they selected heuristic answer. Consequently, the fluency of information processing was lower and FOD was higher.

Contrary to our hypothesis, there were no differences in FOCon and FOD between heuristic and correct answers in non-linear problems. In order to provide correct answer in non-linear problems, students have to detect conflict between heuristic answer and logical principles, and afterwards they have to successfully resolve this conflict (De Neys et al., 2011). Compared to this situation, it can be assumed that when students selected heuristic answer in non-linear problems, they detected conflict, but perhaps 1) they engaged in finding alternative cognitive schema to resolve this conflict, but this schema was not available, or 2) they did not engage in finding alternative schema and immediately after detecting conflict, they relied on appealing heuristic answer. Our results regarding response time are not straightforward and support both assumptions. More precisely, response time for heuristic answers in non-linear problems did not differ from response time for correct answers in linear problems and from response time for correct answers in non-linear problems. It seems that differences in response time for these three answers were small and the response time for heuristic answers in non-linear problems was ranked in the middle (i.e., between correct answers in linear and non-linear problems). Given the findings that conflict detection increases response time (De Neys \& Glumicic, 2008), we can assume that for situations in which students selected heuristic answer in non-linear problems, conflict detection per se increases response time. However, conflict detection is cognitively undemanding process (Fanssens \& De Neys, 2009), so this increase was not sufficient to make a significant difference from generating 
correct answer in linear problems. Nevertheless, our research cannot clarify the reasons for students' reliance on heuristic answers in non-linear problems, so further research is needed.

Response time is used as a measure of fluency of information processing (Baayen \& Milin, 2015; De Neys, 2006; Thompson et al., 2011), and the fluency of information processing is assumed to be the most important determinant of metacognitive experiences (Koriat et al., 2004; Thompson \& Morsanyi, 2012). In the present study, similar to previous research on conflict detection (e.g., Bonner \& Newell, 2010), the slowest response time was in situations in which students provided correct answer in non-linear problems. This lower fluency could have resulted in lower FOCon and higher FOD for non-linear problems in which students selected correct answer than for linear problems in which they selected correct answer. That is, both answers were normatively correct, but in linear problems correct answers were generated fluently because they were congruent with linearity heuristic, while in non-linear problems they were generated after conflict resolution and engagement of the analytic Type 2 processing (i.e., less fluently).

Problems' familiarity and comprehensibility did not differ when correct answers in linear problems, correct answers in non-linear problems, and heuristic answers in non-linear problems were selected. Previous research demonstrated that familiarity is a cue for confidence ratings and that in familiar problems participants have higher FOCon than in abstract problems, irrespective of the levels of logical performance (Markovits, Thompson, \& Brisson, 2015). Given that students had higher FOCon for correct answers in linear problems than correct and heuristic answers in non-linear problems, it could have been expected that students would have higher FOF in former situation than in the two latter situations. However, in our study, both linear and non-linear problems were moderately familiar and very comprehensible, which could be the result of students' exposure to linear and non-linear problems during mathematical education. That is, we used problems that are part of students' formal education, so they were equally familiar and FOF could not serve as an important cue for confidence ratings. Dual meta-representational model (Markovits et al., 2015) suggests that evaluations and confidence ratings of familiar content are based on knowledge-based cues, so perhaps these cues determined differences in FOCon and FOD in our study. Nevertheless, this assumption should be explored in further research.

\section{Metacognitive Experiences, Response Time, and the Type of Answer}

When students selected "none of the answers" in linear and non-linear problems, they had lower FOCon, and estimated those problems as less familiar and comprehensible compared to problems in which they selected correct answer. Moreover, students had higher FOCon, FOF, and FOCom in non-linear problems when heuristic answer was selected than when "none of the answers" was selected. 
Finally, FOD for non-linear problems in which students selected "none of the answers" was higher compared to non-linear problems in which they selected correct and heuristic answer.

Apparently, students did not understand problems in which they selected "none of the answers". They probably were not guessing when they selected "none of the answers" because response time for selecting this answer was higher than for selecting correct answer or distractor in both linear and non-linear problems. Given that analytical Type 2 processing is time-consuming (De Neys, 2006), it can be assumed that students analysed non-linear problems in which they selected "none of the answers", but they could not find an adequate answer. It seems that "none of the answers" had a similar function as "don't know" answer in Ackerman's (2014; Experiment 4 and 5) research. In that research, it was obtained that people, after effortful attempts to find an adequate answer, selected "don't know" because they did not find an answer which satisfied their subjective confidence criterion. As the Diminishing Criterion Model suggests (Ackerman, 2014), people invest time and effort in order to meet their subjective confidence criterion. With prolonged time, this criterion diminishes and at last, people accept the answer, which satisfies their lower confidence criterion. However, when people have "don't know" or "none of the answers" option, they will use it to reject these low-confidence answers. Consequently, confidence is lower and response time is longer when people select "don't know" or "none of the answers". Future research is needed to disentangle why students do not find an adequate solution and select "none of the answers" (e.g., lack of concentration or motivation and poor mathematical knowledge).

\section{Limitations, Future Directions, and Conclusions}

There are a few limitations of the current study. Firstly, participants were students from only one secondary school in Zagreb, so the generalizability of our findings is limited. Secondly, response time was an imprecise measure of the fluency of information processing, which consisted of reading and solving time. Moreover, we cannot prove that students actually thought about problems, so other methods would be useful to implement, such as moving window procedure (De Neys \& Glumcic, 2008), or measuring inspection time by using mouse pointing at answers which student is thinking about (Evans, 1996). Consequently, we can only speculate that heuristic answers were more fluently processed than correct answers in nonlinear problems. Thirdly, we used only one measure of Type 2 processing operationalized as answer accuracy, but other measures such as answer change would be more appropriate (Thompson et al., 2011). Therefore, two-answer paradigm, proposed by Thompson (2009), would give additional insight into the relation between the illusion of linearity and metacognitive experiences. Fourthly, response time is not an exact measure of information processing (Baayen \& Milin, 2015; Houlihan, Campbell, \& Stelmack, 1994), so more precise estimations of the speed of information processing are needed. Finally, measures of cognitive ability or 
thinking style, such as rational-intuitive thinking, are important predictors of heuristic answers (Shiloh, Salton, \& Sharabi, 2002; West \& Stanovich, 2003). Therefore, it would be worthy to examine how these constructs and mathematical knowledge are related to the illusion of linearity.

Despite these limitations, our research adds to the literature on the illusion of linearity and metacognitive experiences. That is, students detected conflict between heuristic answer and mathematical principles in non-linear problems, but failed to inhibit the appealing heuristic answer. Our results also suggest that metacognitive experiences are affected by the fluency of information processing. Current study demonstrated that the findings of conflict detection studies could be applied to the context of mathematical reasoning and problems that have educational and practical relevance. It seems that in the context of the illusion of linearity, not the monitoring of information processing, but the inhibition of heuristic answer is quite lax. Therefore, future research should reveal how inhibitory system can be improved, in order to boost students' adaptive expertise and preparedness for mathematical, but also everyday problem-solving.

\section{References}

Ackerman, R. (2014). The Diminishing Criterion Model for metacognitive regulation of time investment. Journal of Experimental Psychology: General, 143(3), 1349-1368. doi:10.1037/a0035098

Ackerman, R., \& Thompson, V. A. (2017). Meta-reasoning: Monitoring and control of thinking and reasoning. Trends in Cognitive Sciences, 21(8), 607-617. doi:http://dx.doi.org/10.1016/j.tics.2017.05.004

Alter, A. L., \& Oppenheimer, D. M. (2009). Uniting the tribes of fluency to form a metacognitive nation. Personality and Social Psychology Review, 13(3), 219-235. doi:10.1177/1088868309341564

Baayen, R. H., \& Milin, P. (2015). Analyzing reaction times. International Journal of Psychological Research, 3(2), 12-28.

Bonner, C., \& Newell, B. R. (2010). In conflict with ourselves? An investigation of heuristic and analytic processes in decision making. Memory \& Cognition, 38(2), 186-196.

De Bock, D., Van Dooren, W., Janssens, D., \& Verschaffel, L. (2002). Improper use of linear reasoning: An in-depth study of the nature and the irresistibility of secondary school students' errors. Educational Studies in Mathematics, 50, 311-334. doi:10.1023/ A:1021205413749

De Bock, D., Van Dooren, W., Janssens, D., \& Verschaffel, L. (2007). The illusion of linearity: From analysis to improvement. Berlin: Springer. 
De Bock, D., Verschaffel, L., \& Janssens, D. (1998). The predominance of the linear model in secondary school students' answers of word problems involving length and area of similar plane figures. Educational Studies in Mathematics, 35, 65-83. doi:10.1023/ A:1003151011999

De Bock, D., Verschaffel, L., \& Janssens, D. (2002). The effects of different problem presentations and formulations on the illusion of linearity in secondary school students. Mathematical Thinking and Learning, 4(1), 65-89. doi:10.1207/S15327833 MTL0401_3

De Neys, W. (2006). Automatic-heuristic and executive-analytic processing during reasoning: Chronometric and dual-task considerations The Quarterly Journal of Experimental Psychology, 59(6), 1070-1100. doi:10.1080/02724980543000123

De Neys, W. (2010). Heuristic bias, conflict, and rationality in decision-making. In B. M. Glatzeder, V. Goel, \& A. Müller (Eds.), Towards a theory of thinking (pp. 22-33). Berlin: Springer-Verlag.

De Neys, W. (2012). Bias and conflict: A case for logical intuitions. Perspectives on Psychological Science, 7(1) 28-38. doi:10.1177/1745691611429354

De Neys, W. (2014). Conflict detection, dual processes, and logical intuitions: Some clarifications. Thinking \& Reasoning, 20(2), 169-187. doi:10.1080/13546783.2013. 854725

De Neys, W., Cromheeke, S., \& Osman, M. (2011). Biased but in doubt: Conflict and decision confidence. PLoS ONE, 6(1), e15954. doi:10.1371/ journal.pone.0015954

De Neys, W., \& Glumicic, T. (2008). Conflict monitoring in dual process theories of thinking. Cognition, 106(3), 1248-1299. http://dx.doi.org/10.1016/j.cognition.2007.06.002

De Neys, W., Moyens, E., \& Vansteenwegen, D. (2010). Feeling we're biased: Autonomic arousal and reasoning conflict. Cognitive, Affective, \& Behavioral Neuroscience, 10(2), 208-216.

Ebersbach, M., Van Dooren, W., Goudriaan, M. N., \& Verschaffel, L. (2010). Discriminating non-linearity from linearity: Its cognitive foundations in five-year-olds. Mathematical Thinking and Learning, 12(1), 4-19. doi:10.1080/10986060903465780

Efklides, A. (2002). The systemic nature of metacognitive experiences: Feelings, judgments, and their interrelations. In M. Izaute, P. Chambres, \& P.-J. Marescaux (Eds.), Metacognition: Process, function, and use (pp. 19-34). Dordrecht, The Netherlands: Kluwer.

Efklides, A. (2006). Metacognition and affect: What can metacognitive experiences tell us about the learning process? Educational Research Review, 1, 3-14. doi:10.1016/j. edurev.2005.11.001

Efklides, A. (2009). The role of metacognitive experiences in the learning process. Psicothema, 21(1), 76-82.

Efklides, A. (2014). How does metacognition contribute to the regulation of learning? An integrative approach. Psychological Topics, 23(1), 1-30. 
Efklides, A., Samara, A., \& Petropoulou, M. (1999). Feeling of difficulty: An aspect of monitoring that influences control. European Journal of Psychology of Education, 14, 461-476. doi:10.1007/BF03172973

Esteley, C. B., Villarreal, M. E., \& Alagia, H. R. (2010). The overgeneralization of linear models among university students' mathematical productions: A long-term study. Mathematical Thinking and Learning, 12(1), 86-108. doi:10.1080/ 10986060903465988

Evans, J. St. B. T. (1996). Deciding before you think: Relevance and reasoning in the selection task. British Journal of Psychology, 87(2), 223-240. doi:10.1111/j.2044-8295.1996. tb02587.x

Evans, J. St. B. T., \& Stanovich, K. E. (2013). Dual-process theories of higher cognition: Advancing the debate. Perspectives on Psychological Science, 8(3), 223-241. doi:10.1177/1745691612460685

Franssens, S., \& De Neys, W. (2009). The effortless nature of conflict detection during thinking. Thinking \& Reasoning, 15(2), 105-128.

Flavell, J. H. (1979). Metacognition and cognitive monitoring - A new era of cognitivedevelopmental inquiry. American Psychologist, 34, 906-911. doi:10.1037/0003-066X. 34.10.906

Frank, D. J., \& Kuhlmann, B. G. (2017). More than just beliefs: Experience and beliefs jointly contribute to volume effects on metacognitive judgments. Journal of Experimental Psychology: Learning, Memory, and Cognition, 43(5), 680-693. doi:10.1037/ xlm0000332

Gillard, E., Van Dooren, W., Schaeken, W., \& Verschaffel, L. (2009). Proportional reasoning as a heuristic-based process time constraint and dual problem considerations. Experimental Psychology, 56(2), 92-99. doi:10.1027/1618-3169.56.2.92

Hertwig, R., Herzog, S. M., Schooler, L. J., \& Reimer, T. (2008). Fluency heuristic: A model of how the mind exploits a by-product of information retrieval. Journal of Experimental Psychology: Learning, Memory, and Cognition, 34(5), 1191-1206. doi:10.1037/ a0013025

Houlihan, M., Campbell, K., \& Stelmack, R. M. (1994). Reaction time and movement time as measures of stimulus evaluation and answer processes. Intelligence, 18(3), 289-307. doi:10.1016/0160-2896(94)90031-0

Kahneman, D., \& Frederick, S. (2002). Representativeness revisited: Attribute substitution in intuitive judgement. In T. Gilovich, D. Griffin, \& D. Kahneman (Eds.), Heuristics and biases: The psychology of intuitive judgement (pp. 49-81). New York, NY, US: Cambridge University Press.

Koriat, A., Bjork, R. A., Sheffer, L., \& Bar, S. K. (2004). Predicting one's own forgetting: The role of experience-based and theory-based processes. Journal of Experimental Psychology: General, 133, 643-656. http://dx.doi.org/10.1037/0096-3445.133.4.643 
Lerkkanen, M.-K., Rasku-Puttonen, H., Aunola, K., \& Nurmi, J-E. (2005). Mathematical performance predicts progress in reading comprehension among 7-year olds. European Journal of Psychology of Education, 20(2), 121-137. doi:10.1007/BF03173503

Markovits, H., Thompson, V. A., \& Brisson, J. (2015). Metacognition and abstract reasoning. Memory \& Cognition, 43(4), 681-693.

Mueller, M. L., Dunlosky, J., \& Tauber, S. K. (2016). The effect of identical word pairs on people's metamemory judgments: What are the contributions of processing fluency and beliefs about memory? Quarterly Journal of Experimental Psychology: Human Experimental Psychology, 69, 781-799. doi:10.1080/17470218.2015.1058404

Narens, L., Jameson, K. A., \& Lee, V. A. (1994). Subthreshold priming and memory monitoring. In J. Metcalfe \& A. P. Shimamura (Eds.), Metacognition: Knowing about knowing (pp. 71-92). Cambridge, MA, US: The MIT Press.

Nelson, T. O., \& Narens, L. (1994). Why investigate metacognition? In J. Metcalfe \& A. Shimamura (Eds.), Metacognition: Knowing about knowing (pp. 1-25). Cambridge, MA: Bradford.

Norman, E., Price, M. C., \& Duff, S. (2010). Fringe consciousness: A useful framework for clarifying the nature of experience-based metacognitive feelings. In A. Efklides \& P. Misailidi (Eds.), Trends and prospects in metacognition research (pp. 63-80). New York: Springer.

Reber, R., \& Greifeneder, R. (2017). Processing fluency in education: How metacognitive feelings shape learning, belief formation, and affect. Educational Psychologist, 52(2), 84-103. doi:10.1080/00461520.2016.1258173

Rose, H., \& Betts, J. R. (2001). Math matters: The links between high school curriculum, college graduation, and earnings. San Francisco, CA: Public Policy Institute of California.

Shiloh, S., Salton, E., \& Sharabi, D. (2002). Individual differences in rational and intuitive thinking styles as predictors of heuristic answers and framing effects. Personality and Individual Differences, 32, 415-429. doi:10.1016/S0191-8869(01)00034-4

Shynkaruk, J. M., \& Thompson, V. A. (2006). Confidence and accuracy in deductive reasoning. Memory \& Cognition, 34(3), 619-632.

Simmons, J. P., \& Nelson, L. D. (2006). Intuitive confidence: Choosing between intuitive and nonintuitive alternatives. Journal of Experimental Psychology: General, 135, 409-428. doi:10.1037/0096-3445.135.3.409

Song, H., \& Schwarz, N. (2008). If it's hard to read, it's hard to do: Processing fluency affects effort prediction and motivation. Psychological Science, 19, 986-988. doi:10.1111/j. 467-9280.2008.02189.x

Stanovich, K. E., \& Toplak, M. E. (2012). Defining features versus incidental correlates of Type 1 and Type 2 processing. Mind \& Society, 11(1), 3-13. doi:10.1007/s11299-0110093-6 
Thompson, V. A. (2009). Dual process theories: A metacognitive perspective. In J. St. B. T. Evans \& K. Frankish (Eds.), In two minds: Dual processes and beyond (pp. 171-196). Oxford: Oxford University Press.

Thompson, V. A., \& Morsanyi, K. (2012). Analytic thinking: Do you feel like it? Mind \& Society, 11(1), 93-105. doi:10.1007/s11299-012-0100-6

Thompson, V. A., Prowse Turner, J., \& Pennycook, G. (2011). Intuition, reason and metacognition. Cognitive Psychology, 63, 107-140. doi:10.1016/j.cogpsych.2011.06. 001

Thompson, V. A., Prowse Turner, J. A., Pennycook, G., Ball, L. J., Brack, H., Ophir, Y., \& Ackerman, R. (2013). The role of answer fluency and perceptual fluency as metacognitive cues for initiating analytic thinking. Cognition, 128(2), 237-251. doi:10.1016/j.cognition.2012.09.012

Touroutoglou, A., \& Efklides, A. (2010). Cognitive interruption as an object of metacognitive monitoring: Feeling of difficulty and surprise. In A. Efklides \& P. Misailidi (Eds.), Trends and prospects in metacognition research (pp. 171-208). New York: Springer.

Van Dooren, W., De Bock, D., De Bolle, E., Janssens, D., \& Verschaffel, L. (2003). The illusion of linearity: The role of direct versus indirect perimeter and area measures. Mediterranean Journal for Research in Mathematics Education, 2, 1-18.

Van Dooren, W., De Bock, D., Janssens, D., \& Verschaffel, L. (2008). The linear imperative: An inventory and conceptual analysis of students' overuse of linearity. Journal for Research in Mathematics Education, 39(3), 311-342. doi:10.2307/30034972

Verschaffel, L., Greer, B., \& De Corte, E. (2000). Making sense of word problems. Lisse, The Netherlands: Swets \& Zeitlinger.

Vlahović-Štetić, V., Pavlin-Bernardić, N., \& Rajter, M. (2010). Illusion of linearity in geometry: Effect in multiple-choice problems mathematical thinking and learning. Mathematical Thinking and Learning, 12, 54-67. doi:10.1080/10986060903465871

West, R. F., \& Stanovich, K. E. (2003). Is probability matching smart? Associations between probabilistic choices and cognitive ability. Memory \& Cognition, 31(2), 243-251. doi:10.3758/BF03194383 